\title{
Shear Strength of Rockfill, Interfaces and Rock Joints, and their Points of Contact in Rock Dump Design
}

\author{
N.R. Barton Nick Barton and Associates, Norway
}

\begin{abstract}
The peak shear strength of rock joints obtained from direct shear tests, and the peak shear strength of rockfill, as interpreted from large-scale triaxial tests, have common non-linear strength envelopes. An extremely low stress index test for rock joints, the tilt test, with an apparent normal stress as low as $0.001 \mathrm{MPa}$ when sliding occurs, can also be performed to characterise rockfill. However for rockfill or rock dumps, larger samples with relevant particle sizes are desirable. Some full-scale tests at a dam site in Italy, using a 2x2x5 m tilt-shear test, were able to sample the as-compacted-as-built rockfill, with no need for using parallel (model) grading curves with reduced-sized particles. Interfaces between the rockfill or rock dump and eventual rock foundations, can be handled with similar shear strength estimation methods. In each case, a low-stress index test result is extrapolated to full scale and to engineering stress level by related nonlinear strength laws. It is possible to estimate each through inexpensive characterisation. The non-linear, stress-dependent friction angles suggest that large rock dumps with constant slope angle will have strongly reducing factors of safety from top to bottom and from outside to inside.
\end{abstract}

\section{Introduction}

The real contact stress levels are believed to be close to compressive failure where rock joint asperities and rockfill stones are in contact (e.g. Figure 1 for the case of rock joints). Therefore it is perhaps possible to use a common form of constitutive equation for extrapolating the strength measured at very low (index test) normal stress levels, to stress levels of engineering interest, as inside a large rockfill dam, inside a rock dump or under a rock slope formed of jointed rock.
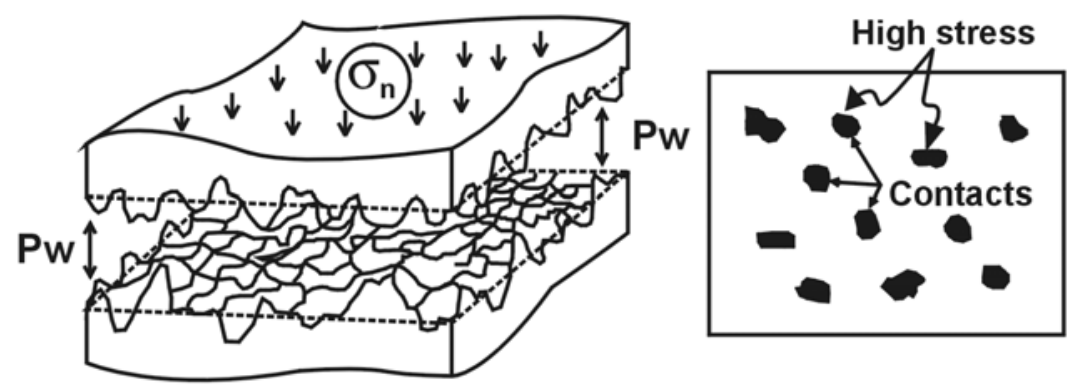

Figure 1 When peak shear strength is approached (joints and rockfill), the actual rock-to-rock contact stress levels are extremely high, due to small contact areas

It is believed that the real ratios of $\sigma_{\mathrm{cn}} / \mathrm{JCS}$ (contact normal stress/joint wall compressive strength, in the case of rock joints) and $\sigma_{\mathrm{cn}} / \mathrm{S}$ (contact normal stress/particle strength,in the case of rockfill) are equal to the ratio $\mathrm{A}_{0} / \mathrm{A}_{1}$ representing the ratio of true contact area/assumed contact area. The terms JCS and S represent the joint compressive strength and the particle strength, respectively. In other words, contact area is a rock strength or particle strength regulated phenomenon at peak strength.

Tilt tests are performed on a regular basis to characterise the roughness of rock joints. A schematic example of tilt testing for rock joints is shown in Figure 2, while a suggested method for testing rockfill at full scale (without needing parallel grading curves) is shown in Figure 3, from Barton and Kjærnsli (1981). 


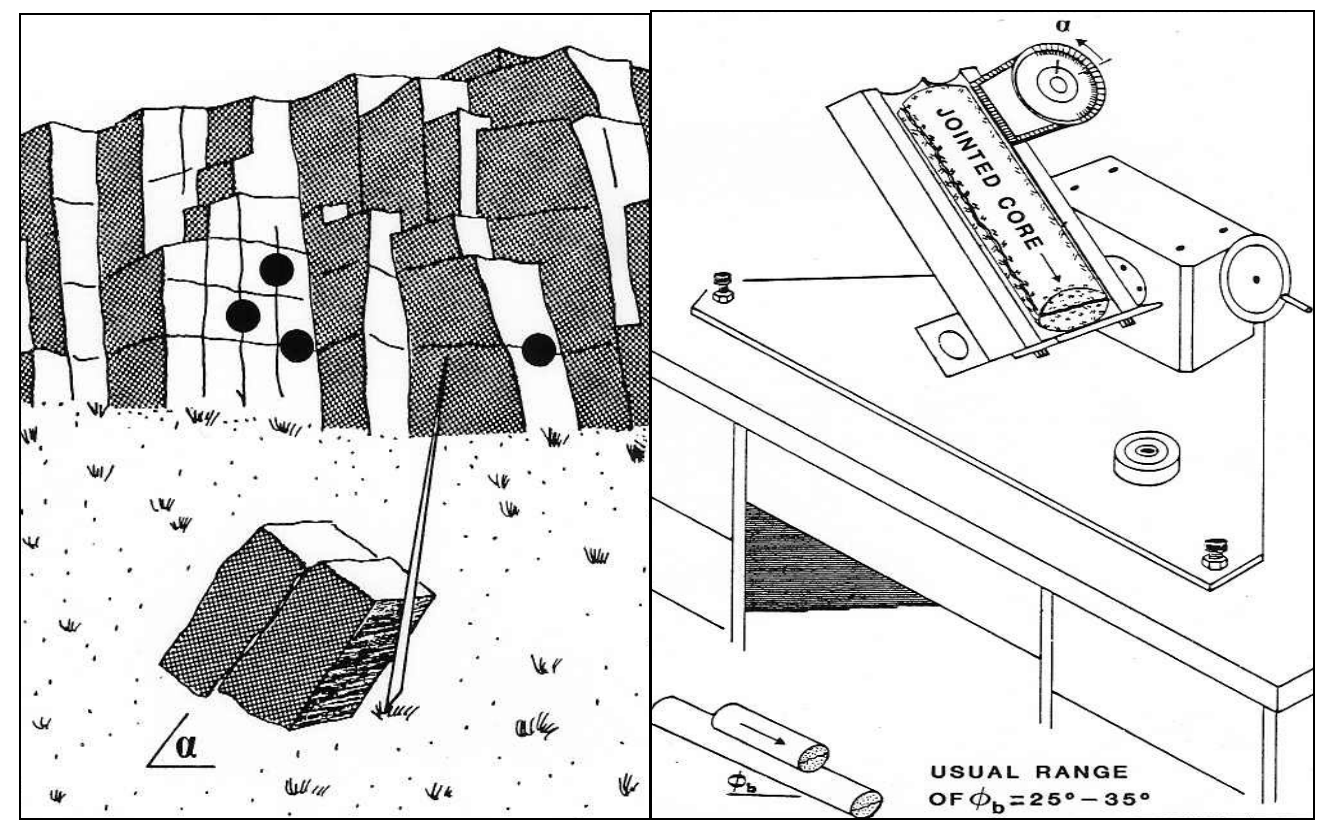

Figure 2 Tilt test (or self-weight gravity shear test) for characterising rock joints. Note measurement of basic friction angle on unweathered, smooth (but not polished) pieces of core

The equation for back-calculating the effective roughness (R) of rockfill particles is shown in Figure 3 (diagram 5). Exactly the same format is used to back-calculate the joint roughness coefficient (JRC) for rock joints:

$$
\operatorname{JRC}=\left(\alpha^{\mathrm{o}}-\varphi_{\mathrm{r}}\right) / \log \left(\mathrm{JCS} / \sigma_{\mathrm{no}}\right)
$$

where $\sigma_{\text {no }}$ represents the very low normal stress acting when sliding occurs between the two halves of a mating rock joint, at tilt angle $\alpha^{\circ}$. In the case of tilt tests on laboratory-scale joint samples, the normal stress is often as low as $0.001 \mathrm{MPa}$. In the case of the $5 \mathrm{~m}$ long tilt test performed at a dam site in Italy (one of 10 such tests using compacted rockfill, filter material or sand), the normal stress was closer to 0.01 or $0.02 \mathrm{MPa}$, resulting from the 1 meter thickness of the upper shear box (Figure 4). 


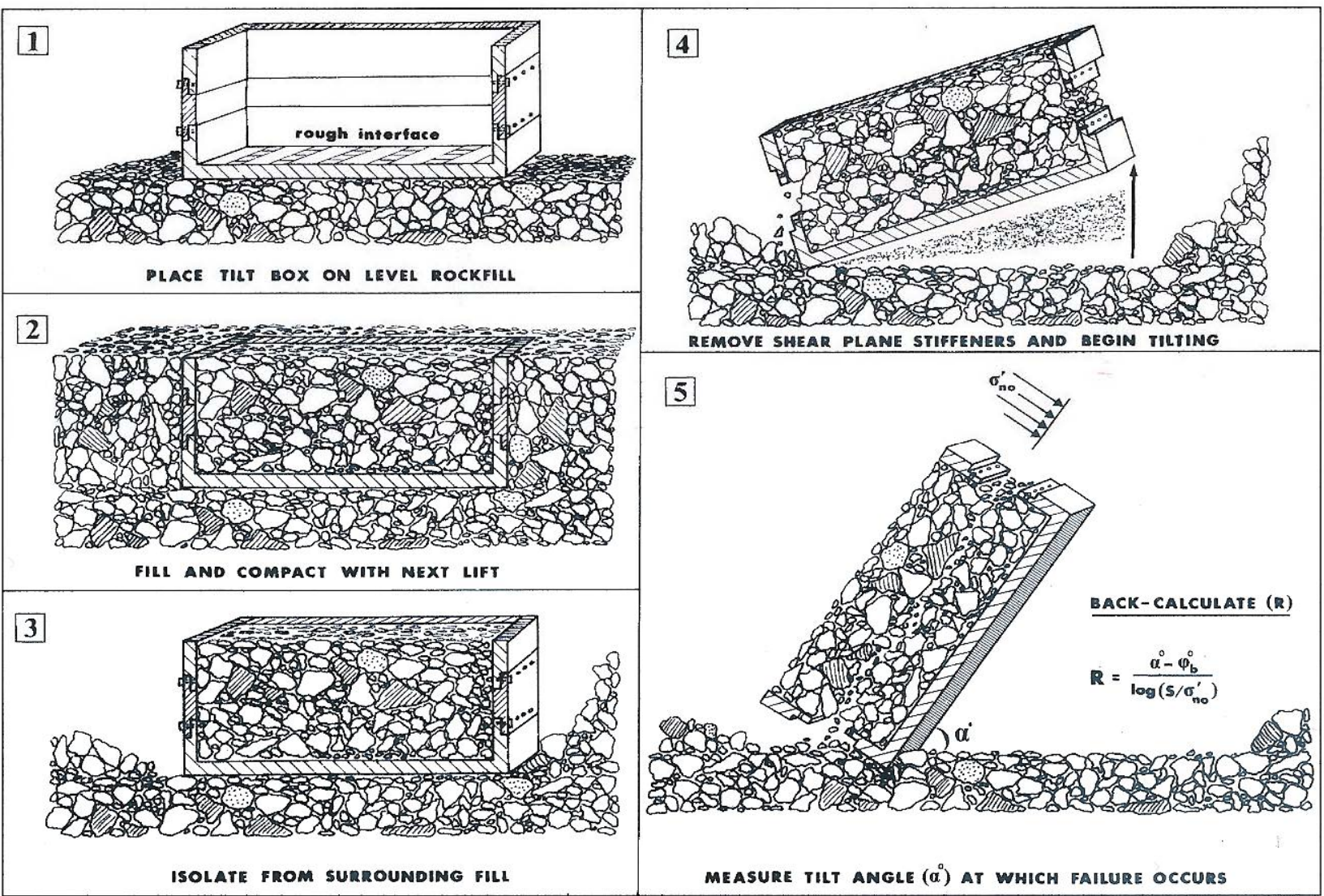

Figure 3 Illustration of the tilt test principle for rockfill (Barton and Kjærnsli, 1981)

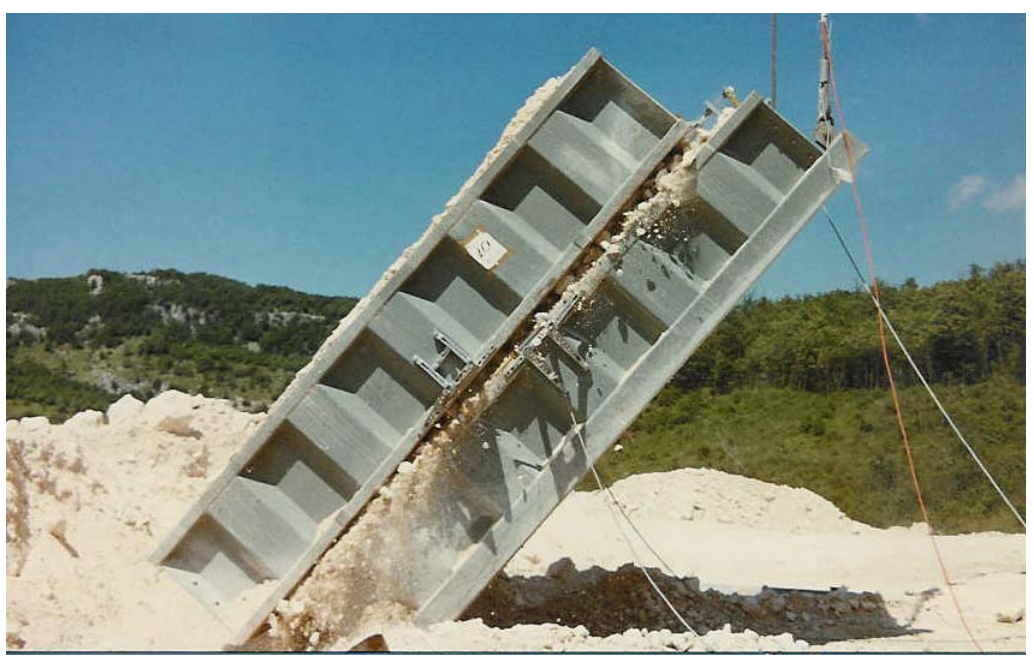

Figure 4 In situ $5 \mathbf{m}$ long rockfill tilt test being performed during dam construction in Italy (Strøm, 1987)

\section{The shear strength of rock joints}

A basic set of peak and residual shear strength laws that are frequently used in rock mechanics are illustrated in Figure 5. In this paper, focus will be on the third equation, due to specific treatment of joint roughness (JRC), joint wall compressive strength (JCS) and residual friction angle $\varphi_{\mathrm{r}}$, as determined from index tests shown in Figure 6. 


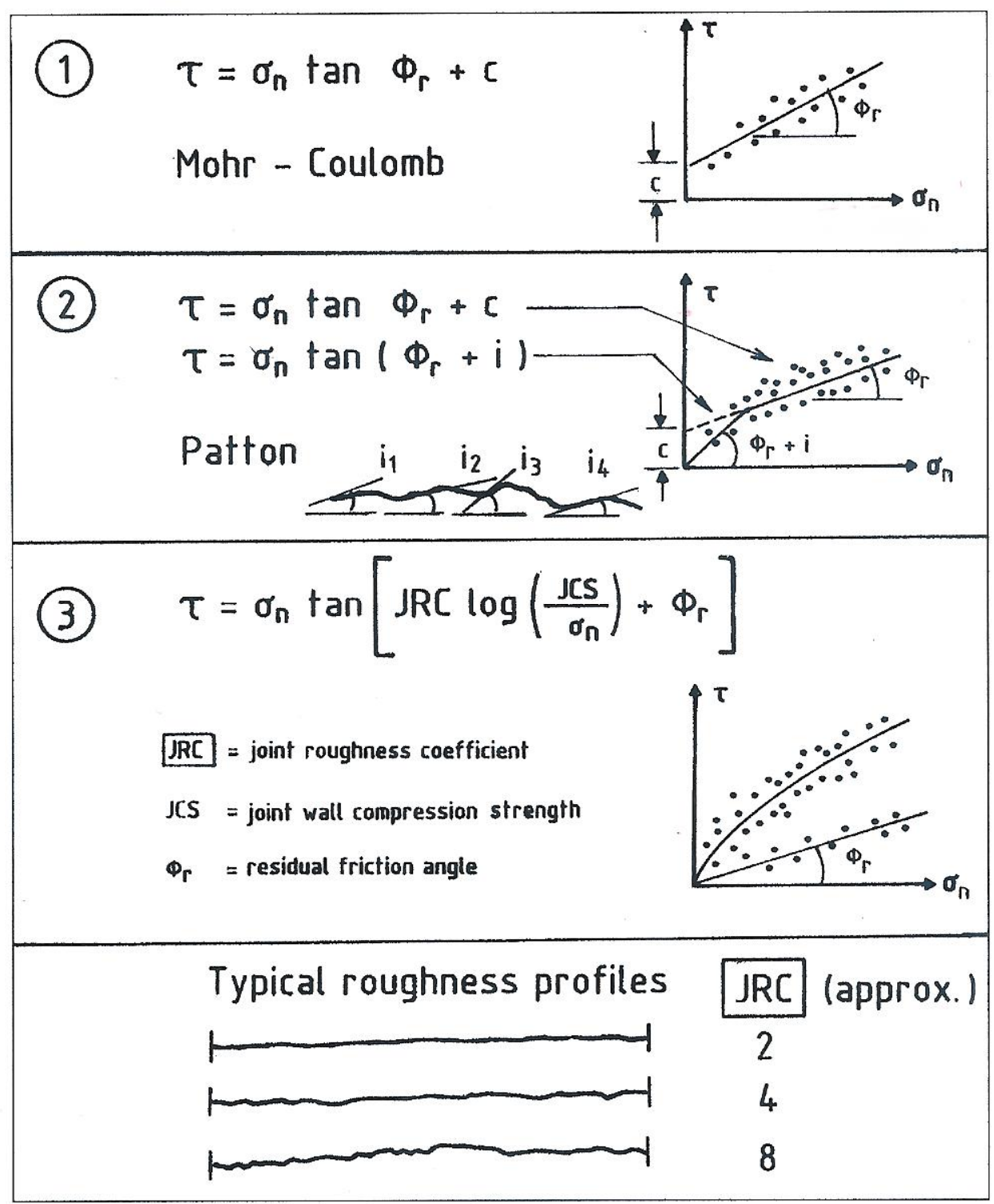

Figure 5 Three shear strength criteria for rock joints: 1 Mohr Coulomb, 2 Patton and 3 Barton. Note that the ' $i$ ' value of Patton is replaced by a roughness term (JRC), and strength/stress ratio $\mathrm{JCS} / \sigma \mathrm{n}$ in the case of the non-linear model 


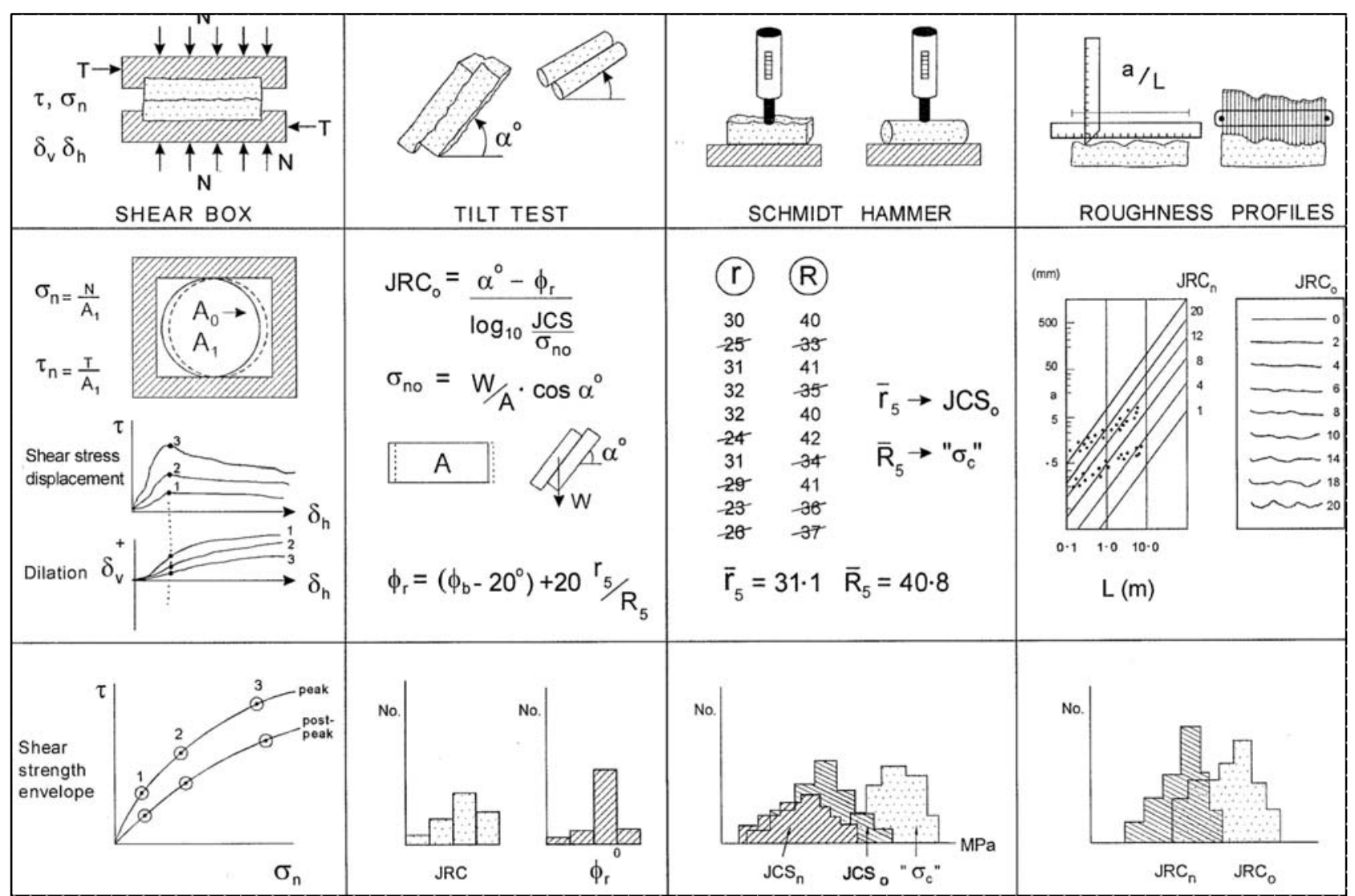

Figure 6 A variety of index tests for characterising the strength components of rock joints, with particular emphasis on roughness (Barton, 1999). Subscripts ' 0 ' and ' $n$ ' in the histograms, refer to laboratory and in situ scale respectively

Examples of peak shear strength measurements for rock joints are shown in Figures 7, 8 and 9. These tests were performed as 130 DST tests on 130 joint samples (not multi-stage testing). The peak strength of each sample was therefore determined.

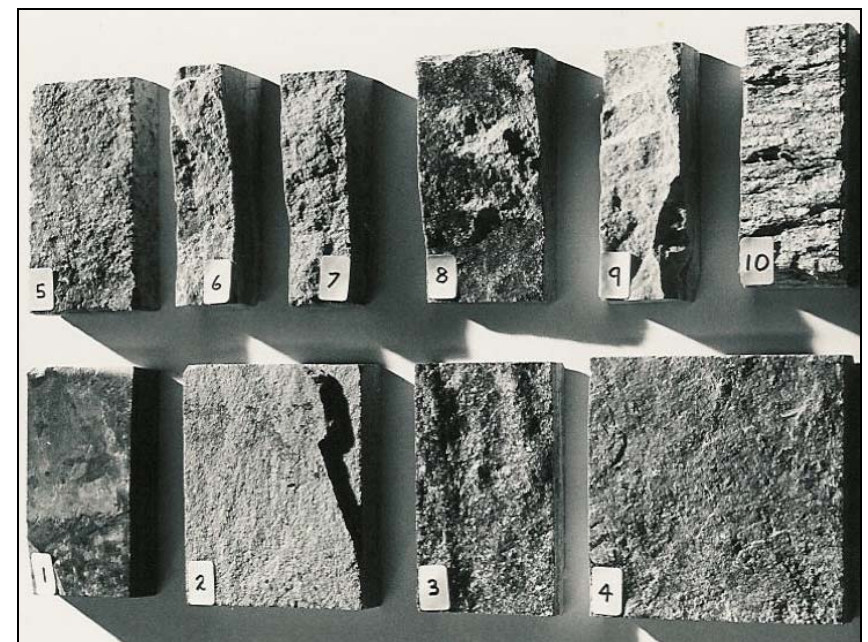

Figure 7 Ten typical (and increasingly rough) joint surfaces, as tested by Barton and Choubey, 1977 (Note that \#10 is an artificial tension fracture in soapstone)

A range of index tests has been developed for estimating the shear strength of rock joints, so that a statistical range of roughness and strength values can be accommodated in design, without the need for an equally large number of direct shear (box) tests, or DST. Besides tilt testing, the index tests illustrated in Figure 6 
also show roughness profiling, which is not only useful for rock joint characterisation, but also useful for interface strength investigations.

It can be noted from Figure 6 that the Schmidt hammer index test is used on rock joints to register what may be a thin 'skin' of weathered rock with lower joint wall strength (JCS) than the 'fresh' rock uniaxial compressive strength $\sigma_{\mathrm{c}}$. This index test can also be used with advantage on rockfill materials, if stones are suitably clamped when small enough to move under the impact of the spring-loaded 'hammer'.

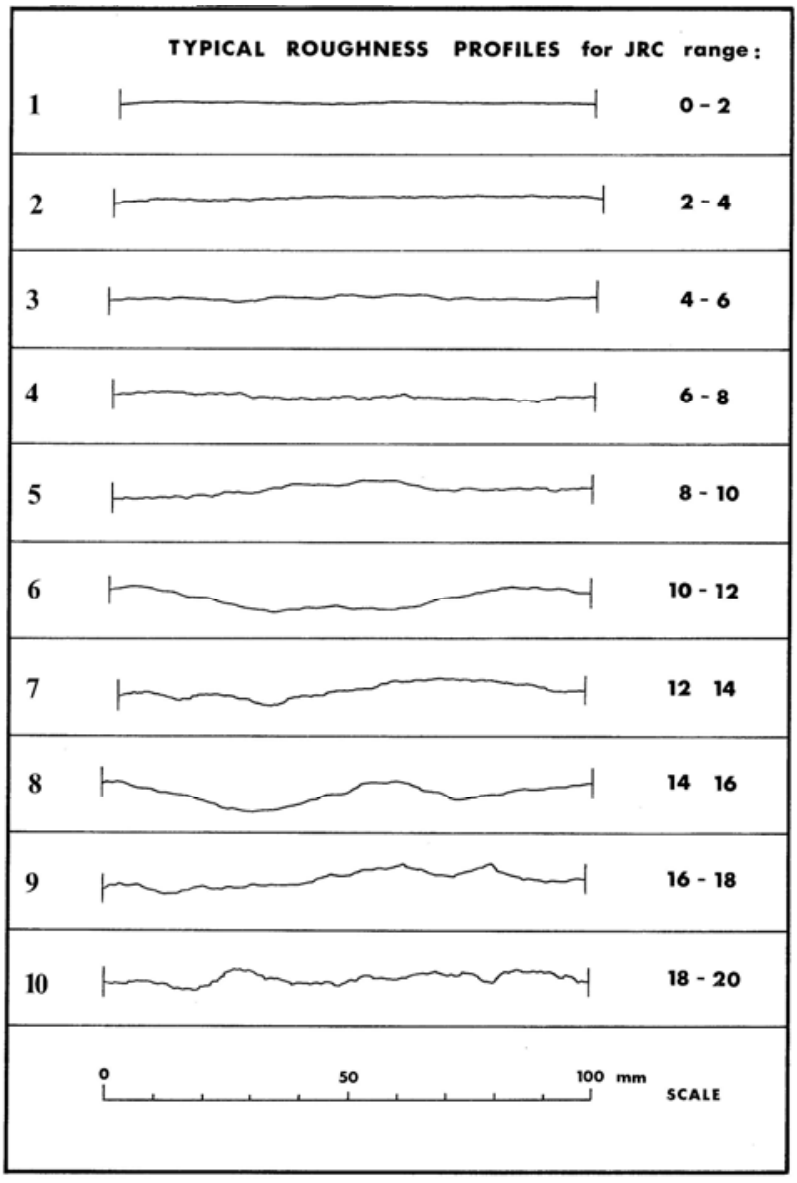

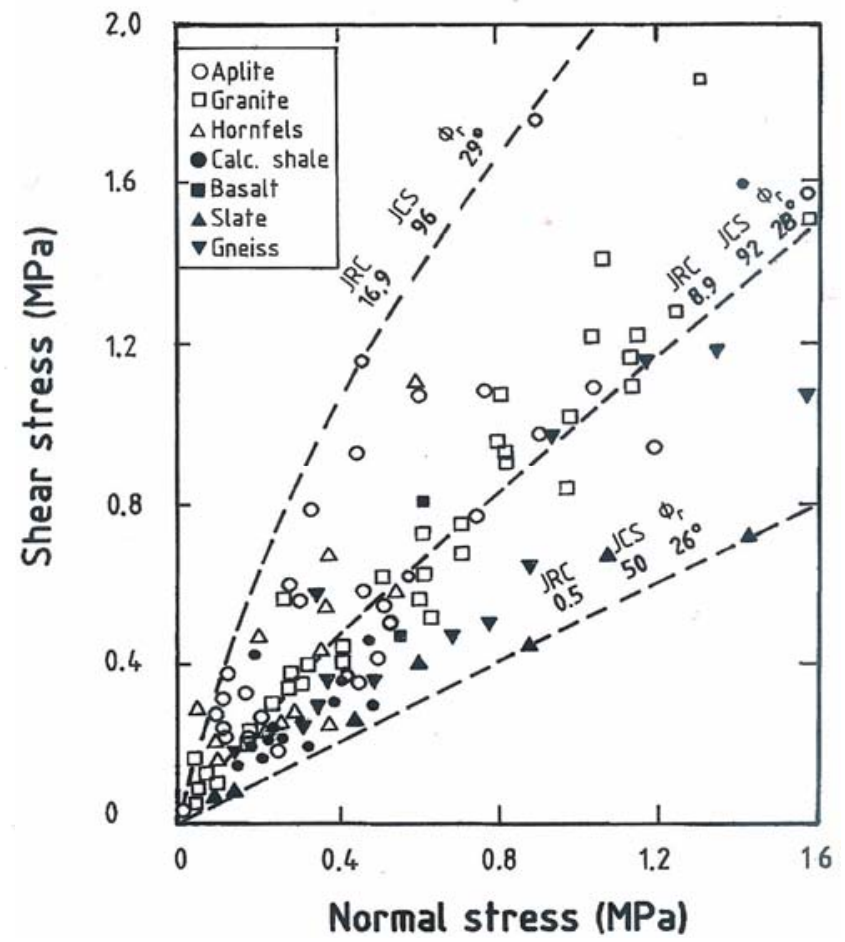

Figure 8 The range of joint roughness profiles tested, and their JRC values, based on the peak shear strength of 130 rock joints. Note the characteristics (JRC, JCS, $\left.\varphi_{r}\right)$ of three example strength envelopes (Barton and Choubey, 1977). The units of JCS are (also) MPa

\section{The shear strength of rockfill as measured}

Leps (1970) is responsible for assembling a significant number of large-scale triaxial shear test data for rockfills of various types. The interpreted peak effective friction angles as a function of the estimated effective normal stress are shown in Figure 9a. We can 'fit' familiar values of JRC and JCS for rock joints (Figure 9b) that closely match the stress-dependent friction angles that (also) describe the shear strength of rockfills. Mid-range JRC values (to correspond to an R-range of about 5 to 10, and low-to-high range JCS values (to correspond to an S-range of about 10 to $100 \mathrm{MPa}$ ) generated by medium weak to medium strong rock are seen to fit the test data. The more conventionally plotted shear stress versus effective stress curves for rockfill, shown in Figure 10 from Marsal (1973), also confirm the similarities of the peak shear strength of rock joints and rockfill. 

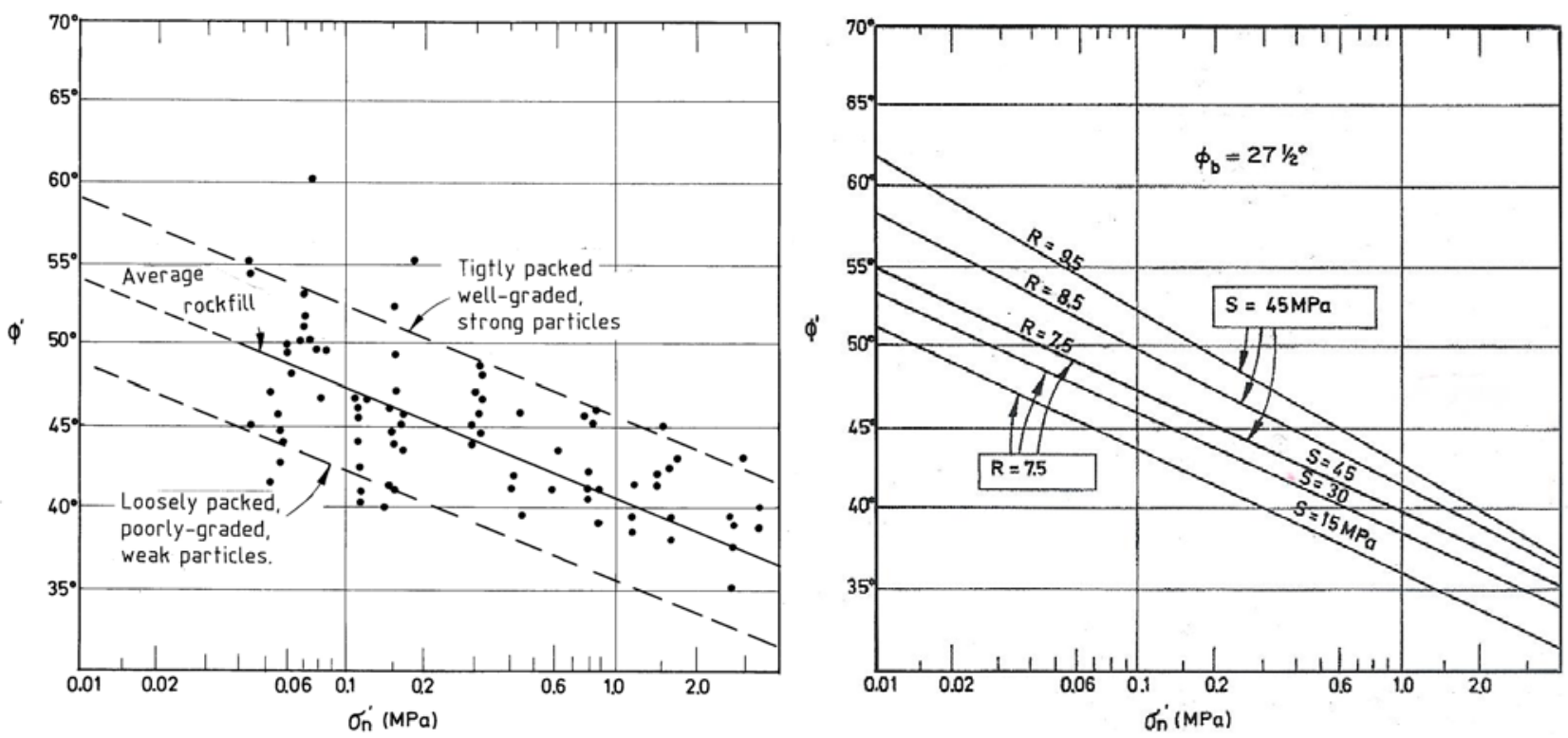

Figure 9 Left: Assembly of peak shear strength data for rockfills, from Leps (1970). Right:

Comparative JRC or R, and JCS or S values used to generate similar gradients to Leps 1970 data for rockfill. $R=5$ to 10 , and $S=10$ to $100 \mathrm{MPa}$ appear to cover the range of strengths assembled by Leps. Less compacted rock dump materials will tend to have lower 'R-values' than the 'tightly-packed' particles, since there will generally be less interlocking

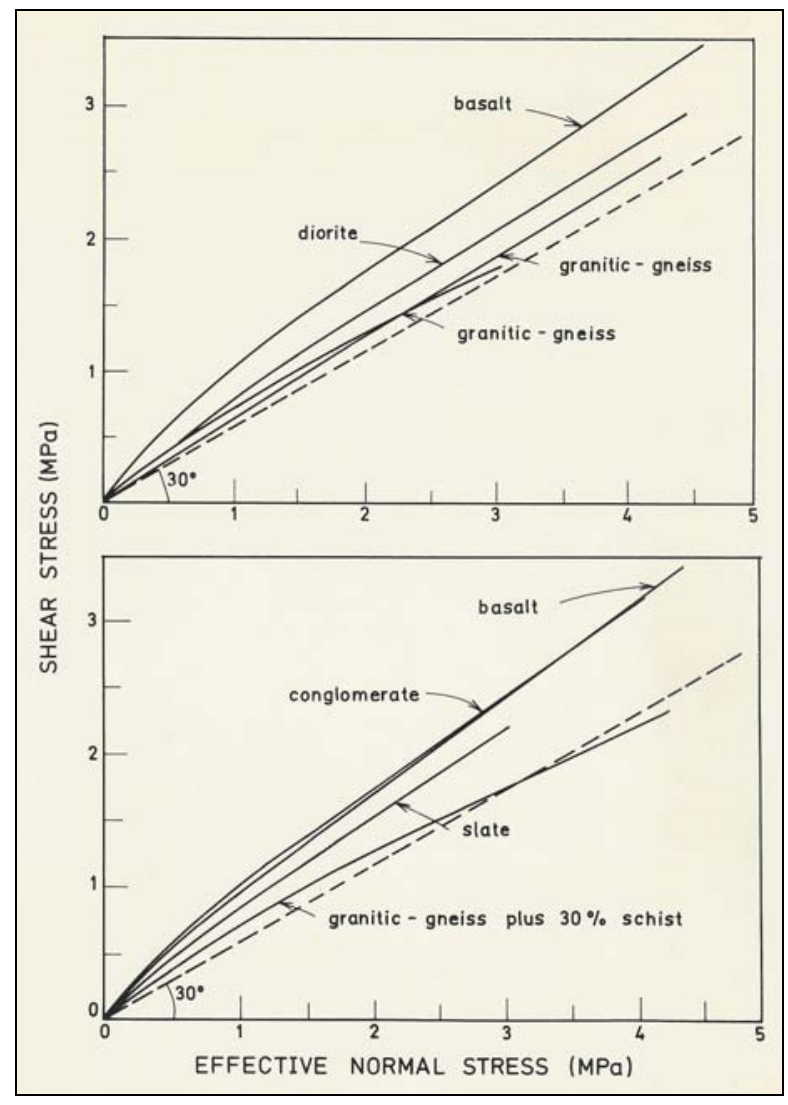

Figure 10 The peak shear strength envelopes for rockfill have remarkable similarity to those for medium rough, medium strength rock joints. Large-scale test data from Marsal (1973) 

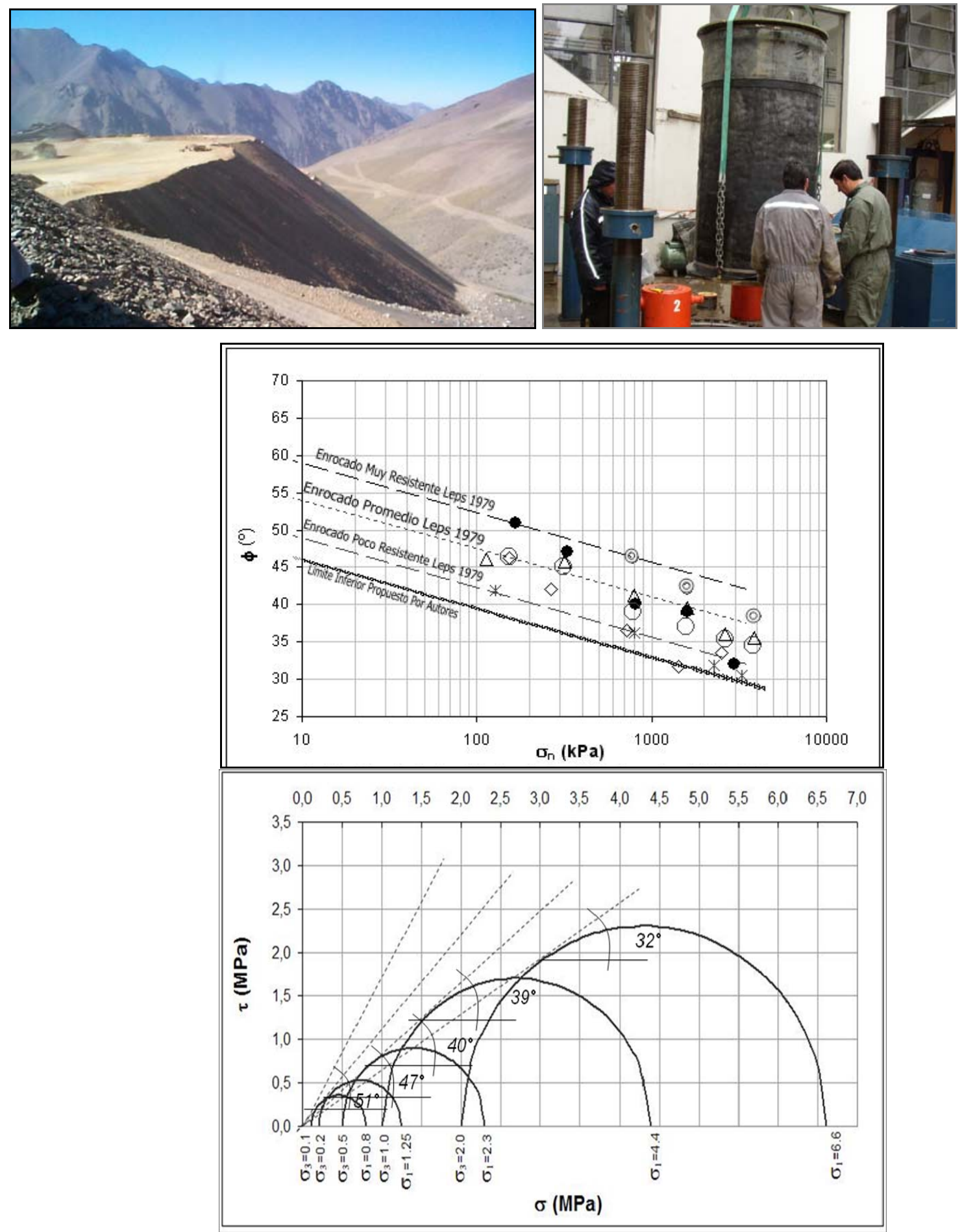

Figure 11 Large rock dumps are a familiar feature of mines in the Chilean Andes. Large-scale triaxial shear tests performed in Chile, with important results (black dots and Mohr circles) showing non-linear stress-dependent friction angles (Linero and Palma (2006), reproduced with kind permission)

The large scale measurement of frictional strength of rock dump materials obtained from mines in the Chilean Andes shown in Figure 11 tend to further reinforce the idea of non-linear stress-dependent friction angles that are likely to apply to rock dumps in general (priv. comm., Sandra Linero, SRK). 


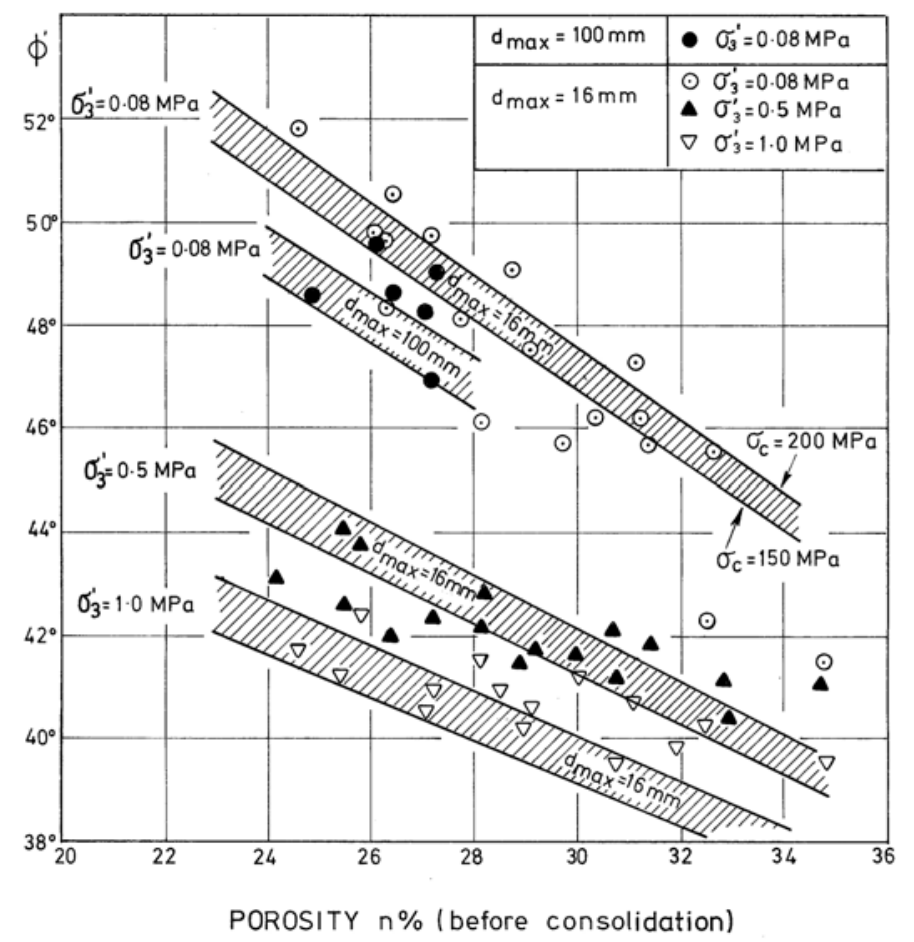

Figure 12 The same non-linearity with effective stress level is seen in large-scale triaxial tests performed at NGI (Strom, 1974, 1975, 1978), with particle size-dependence, rock strength dependence, and porosity effects also indicated

For comparison, Figure 13 shows shear strength envelopes for rock joints that have been generated with the JRC-JCS model introduced in Figure 5. The strongly varying peak dilation angles, part of the reason for the non-linearity, are also shown on each envelope, except at lowest stress, where they may exceed $30^{\circ}$.

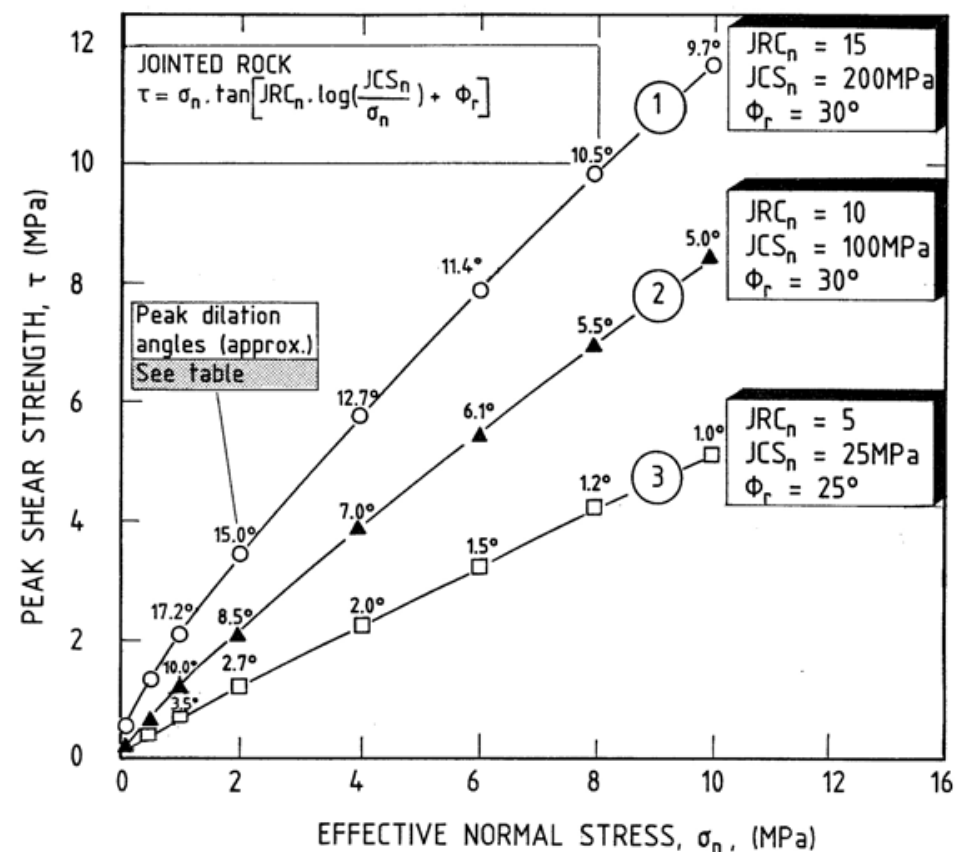

Figure 13 Shear strength envelopes (and peak dilation angles) predicted for rock joints, using the JRC-JCS non-linear model of Figure 5. Rockfill generally lies between curves \#2 and \#3 


\section{Estimating the shear strength of rockfill}

As emphasised in all reports of rockfill shear strength, including Barton and Kjærnsli (1981), the degree of compaction and porosity achieved when building a dam or when preparing relevant laboratory samples is all important. The particle roughness and smoothness is also fundamental. Figure 14 illustrates an empirical scheme developed by the writer, for estimating the likely R-value for rockfills, whether for rounded gravels or for rough quarried rock. The high (relatively uncompacted) porosities in mining rock dumps clearly places such dumps in the middle-to right-hand areas of this diagram, and even sharp angular particles (relevant for waste rock, but perhaps not always for tailings) are unlikely to generate 'R-values' above 5 to 7 , as also suggested in Figure 9.

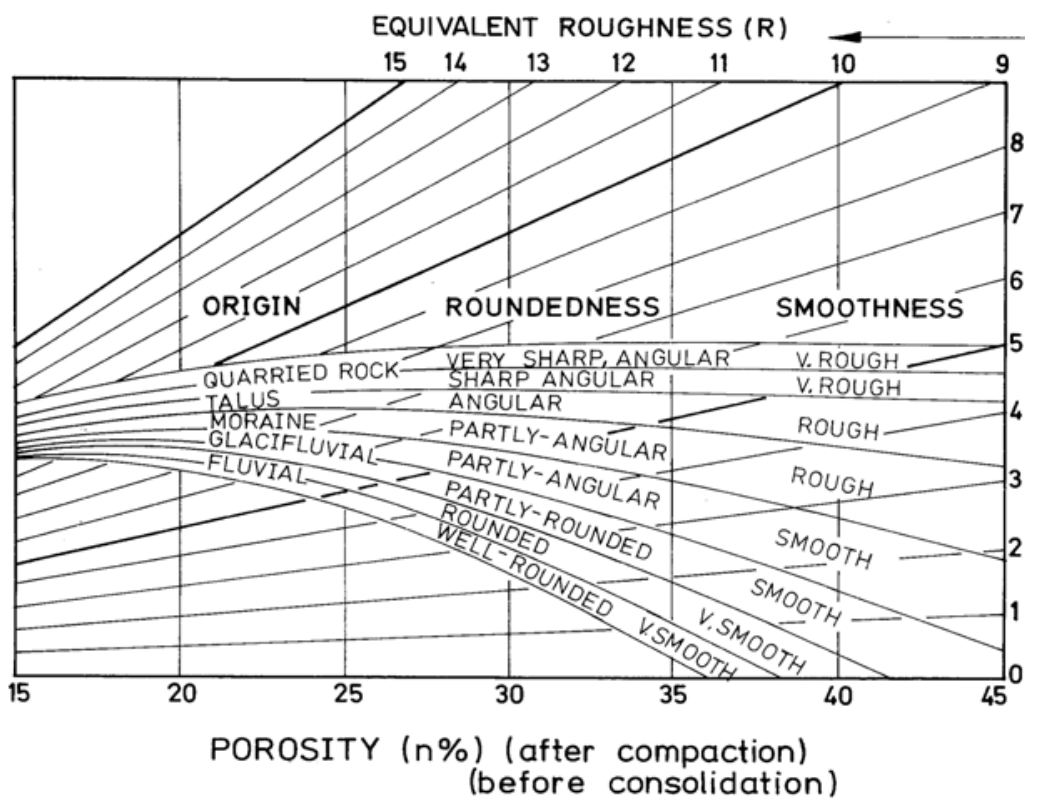

\begin{tabular}{|c|c|c|c|c|}
\hline \multicolumn{3}{|c|}{ EXAMPLES SHOWING DEGREE OF ROUNDEDNESS } \\
\hline $\begin{array}{c}\text { QUARRIED } \\
\text { ROCK }\end{array}$ & TALUS & MORAINE & $\begin{array}{c}\text { GLACIFLUVIAL } \\
\text { MATERIAL }\end{array}$ & $\begin{array}{c}\text { FLUVIAL } \\
\text { MATERIAL }\end{array}$ \\
\hline & & & & \\
\hline
\end{tabular}

Figure 14 An empirical method for estimating the equivalent roughness $R$ of rockfill as a function of porosity and particle origin, roundedness and smoothness. Barton and Kjærnsli (1981)

As a result of the literature survey of numerous rockfill test data, Barton, 1980 and Barton and Kjærnsli, 1981 developed a simple strength factoring scheme for estimating $\mathrm{S}$ as a function of UCS (or $\sigma_{\mathrm{c}}$ ), when particle size $\left(\mathrm{d}_{50}\right)$ varied over a wide range. The points $\mathrm{A}$ and $\mathrm{B}$ in Figure 15 were used to illustrate S-value estimation for a rock with $\mathrm{UCS}=150 \mathrm{MPa}$, when $\mathrm{d}_{50}$ was $23 \mathrm{~mm}(\mathrm{~S} \approx 0.3 \times 150=50 \mathrm{MPa})$ and when $\mathrm{d}_{50}$ was $240 \mathrm{~mm}(\mathrm{~S} \approx 0.2 \times 150=30 \mathrm{MPa})$, in the case of interpreting triaxial strength data. Note the higher factors apparently needed when planar (and large-scale) shear is involved. Friction angles are typically several 
degrees higher (e.g. about $2^{\circ}$ to $4^{\circ}$ ) when plane tests are compared with triaxial tests on the same material. There is noticeably less crushing of particles: hence the two empirical curves in Figure 15.

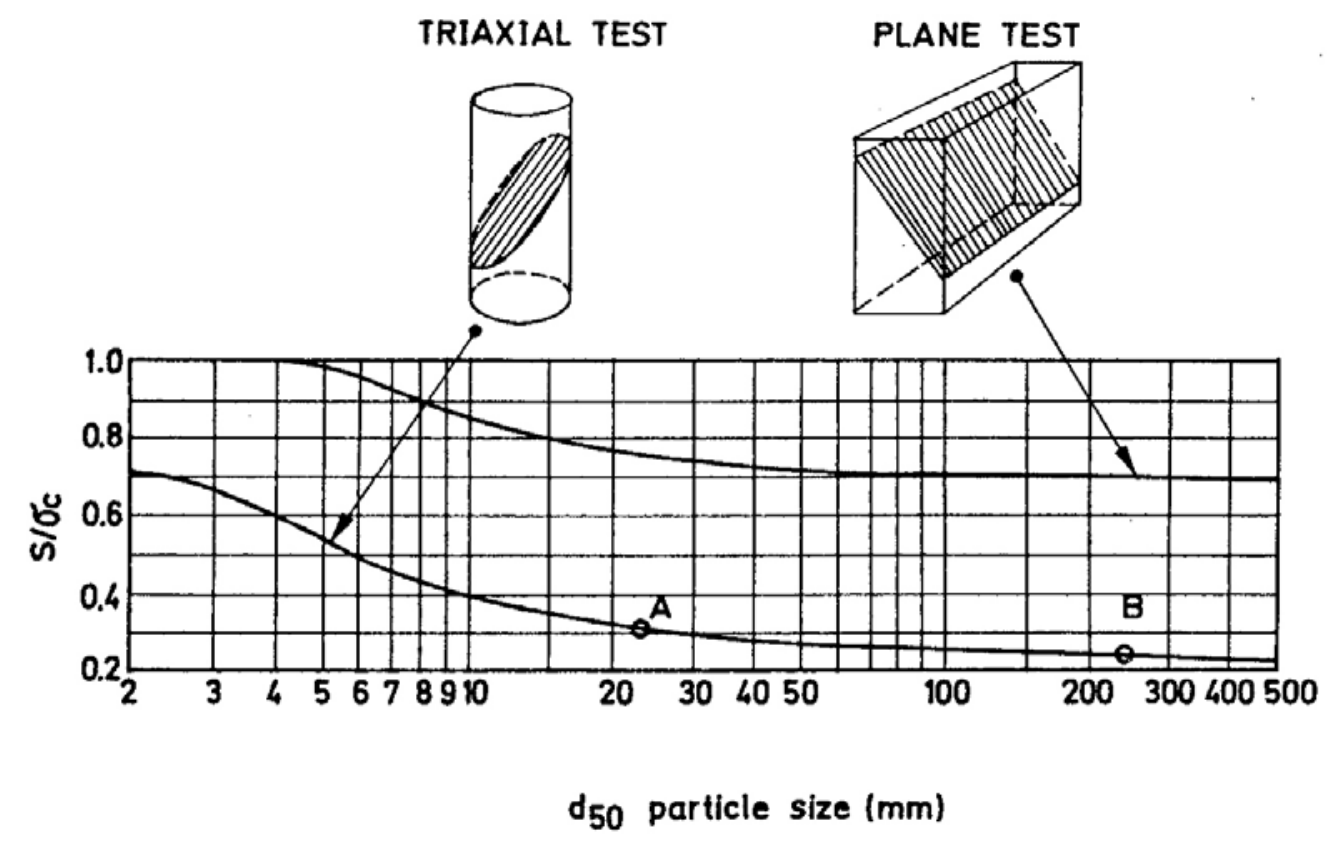

Figure 15 Particle size strongly effects the strength of contacts points in rockfill. Triaxial or plane shear also influences behaviour. Empirical S/UCS reduction factors for estimating $S$ when evaluating equation 3.

\section{$5 \quad$ Interface shear strength}

Interface shear strength, as between a (too smooth) rock foundation and a rockfill dam, seems to be governed by the 'weakest link' rule. If the roughness JRC of the interface, registered by amplitude/length profiling, is too low in relation to particle size $\left(\mathrm{d}_{50}\right)$, the interface strength is controlled by JRC, and sliding occurs along the interface, as along the bottom face of a rock joint. If on the other hand, the interface roughness is sufficient to give good interlock to the rockfill particles, sliding will occur preferentially within the rockfill, in an 'R-controlled' particle smoothness or roughness dependent manner, with influence also of the porosity. A schematic illustration of the interface problem, and (probable) relevant controlling parameters is shown in Figure 16. 


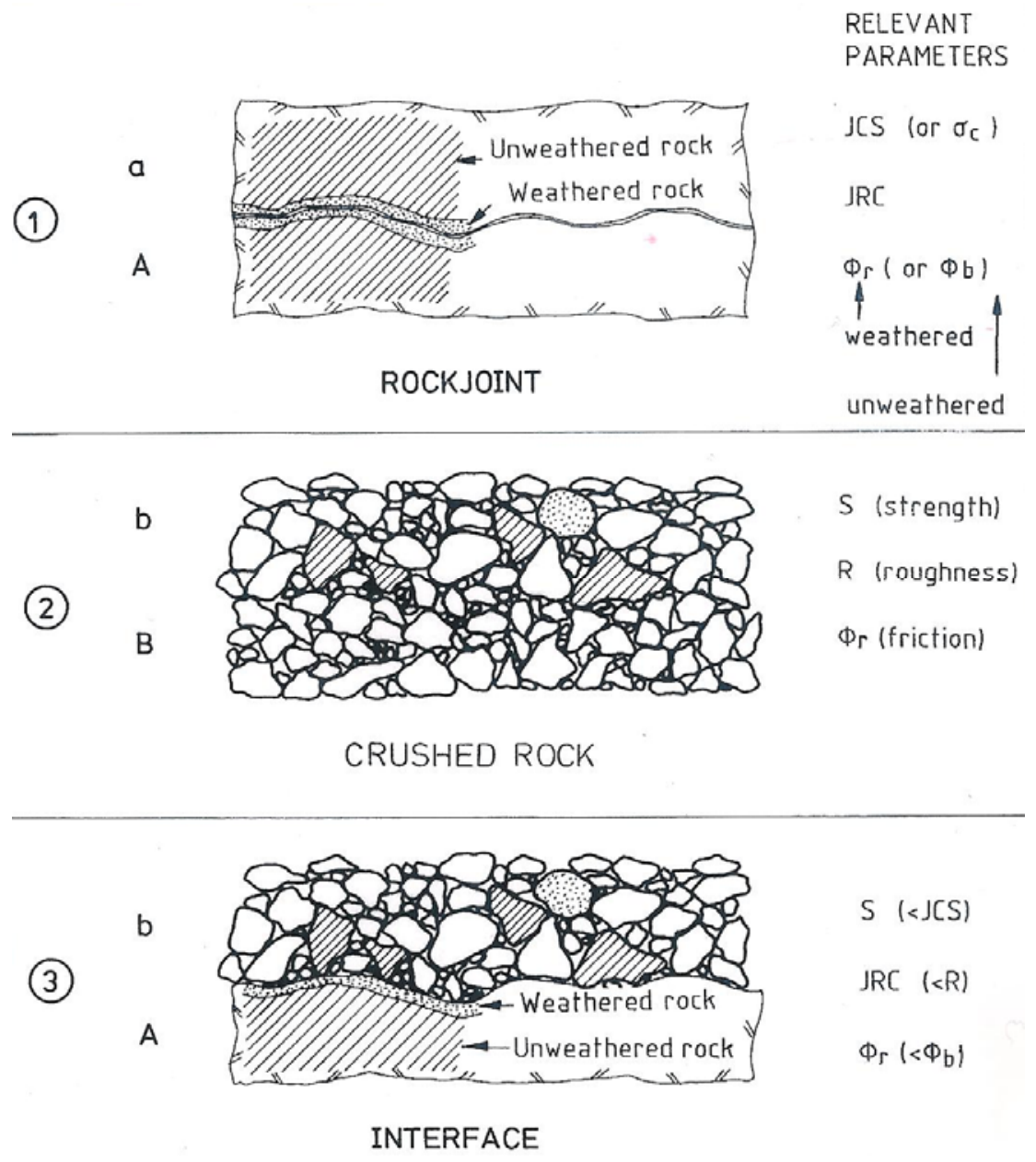

Figure 16 Asperity contact across stressed rock joints, and rockfill inter-particle contact, and rockfill lying on a rock foundation, are each examples of point-contact stress levels that are probably close to compressive failure, when peak shear strength is approached. For this reason the three cases have many points in common, including similar non-linear shear strength envelopes

The peak shear strengths for rock joints, rockfill and interfaces are respectively:

Rock joints:

$$
\tau=\sigma_{n} \tan \left(J R C \log J C S / \sigma_{n}+\phi_{r}\right)
$$

Rockfill:

$$
\tau=\sigma_{n} \tan \left(R \log S / \sigma_{n}+\phi_{r}\right)
$$

Interface:

$$
\tau=\sigma_{n} \tan \left(J R C \log S / \sigma_{n}+\phi_{r}\right)
$$

If the rockfill particles are not weaker than the rock foundation, as assumed in equation 4 , then $\mathrm{S}>\mathrm{JCS}$, and the strength is determined by the weak foundation. In the case of rockfill or waste rock that is freshly blasted, the residual friction angle $\varphi_{r}$ assumed, can (initially) be replaced by $\varphi_{b}$, which is usually a few degrees higher than the weathered value. Conservative, long-term design strength may nevertheless demand the use of $\varphi_{r}$ for 'permanent' rock dumps and rockfill dams, as suggested in all three equations. 


\subsection{R-controlled or JRC-controlled behaviour}

As indicated above, the relative magnitudes of the interface parameters, and their possible contrast to the shear strength of the rockfill, will determine whether the interface (if very rough) causes 'R-controlled' behaviour - meaning preferential failure through the rockfill, or 'JRC-controlled' behaviour, meaning preferential shear along the interface. A review of interface tests, performed by Barton (1980) in response to doubts about the strength of a glacially-smoothed dam foundation in Norway, resulted in the separation of performance identified in Figure 17.
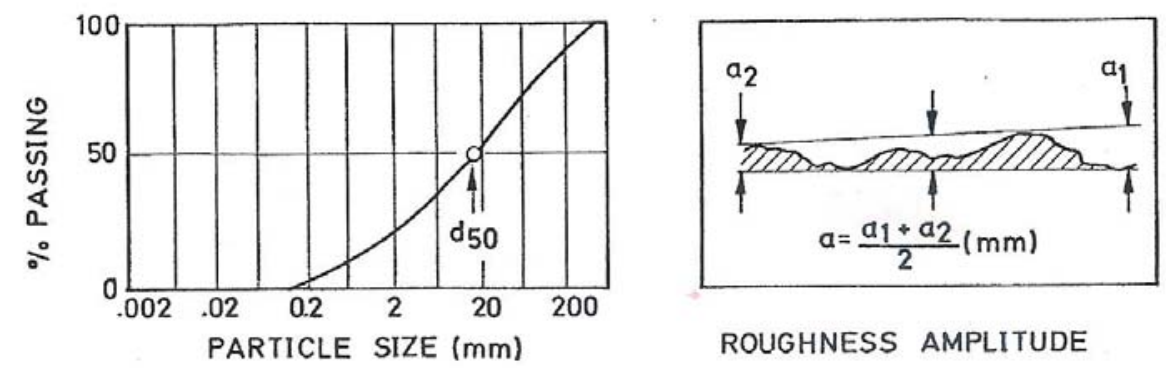

ROUGHNESS AMPLITUDE

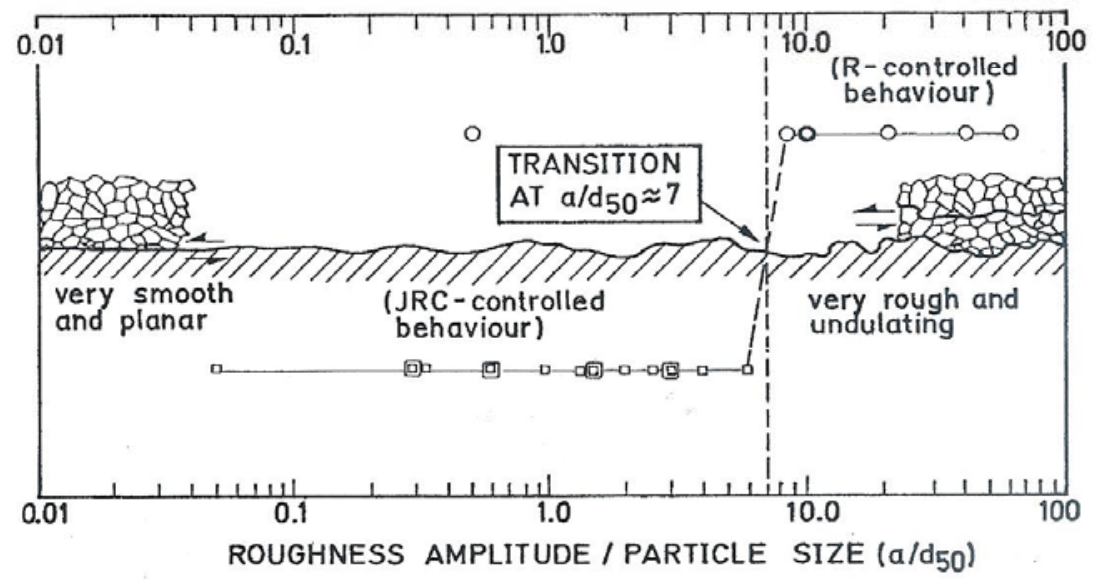

Figure 17 A review of interface shear tests was performed in response to concern over insufficient roughness for the rockfill dam foundation, in the glaciated mountain terrain in Norway. This review resulted in the JRC-R separation seen here. Squares and circles are data points derived from interpretation of relevant tests reported in the literature (Barton, 1980) 

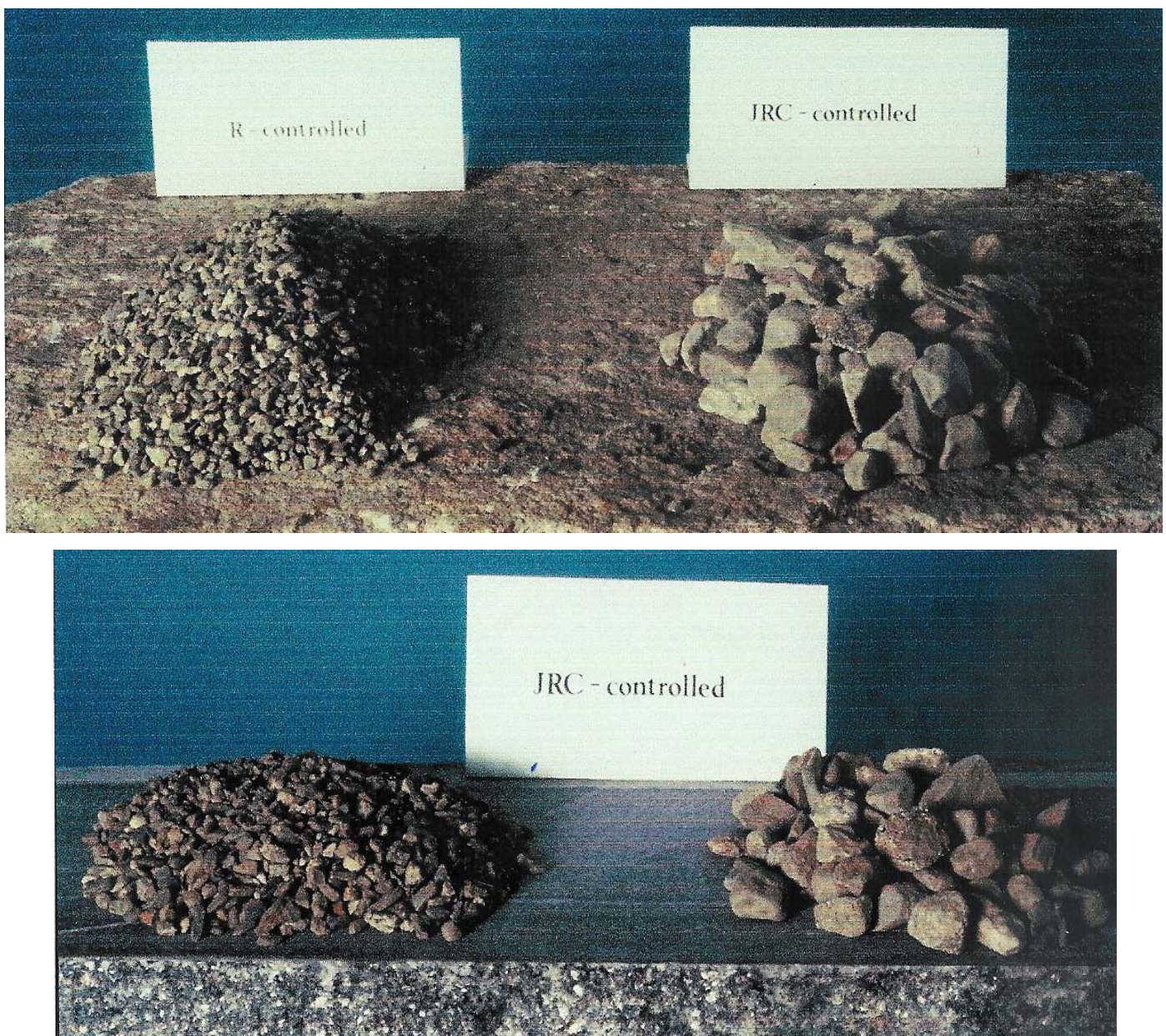

Figure 18 Simple demonstration of the differences between JRC-controlled and R-controlled behaviour, which can also be demonstrated by tilt testing

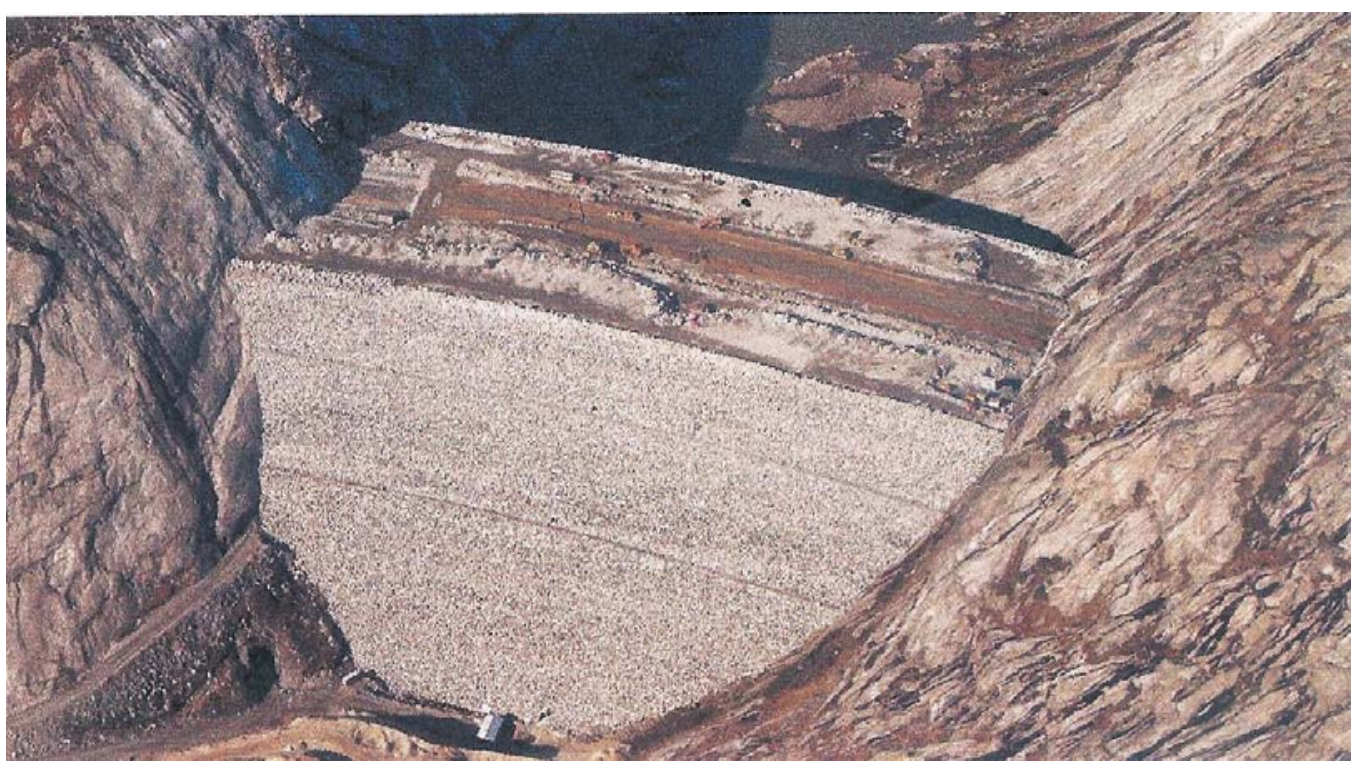

Figure 19 The Svartevann dam in Norway has limited roughness in parts of its steep glaciated abutments. The $a / L$ versus $d_{50}$ ratios for this dam are not known however. These governing parameters were estimated for the Oddatjørn dam in another actually less glaciated location (Barton, 1980) 


\section{Conclusions}

- Rock joints, rockfill (and rock dumps), and interfaces between rock and rockfill have related geotechnical behaviour, because they have 'points-of-contact' in common.

- The common denominator is very high rock-to-rock contact stress at the numerous points of contact.

- The important geotechnical result is a shared tendency for very non-linear, stress-dependent shear strength.

- It is therefore extremely likely that rock dumps of different heights will have widely different factors of safety - if formed with equal slope angles.

\section{References}

Barton, N. and Choubey, V. (1977) The shear strength of rock joints in theory and practice. Rock Mechanics 1/2:1-54. Vienna: Springer.

Barton, N. (1980) Evaluation of shear strength in rockfill and between rockfills and rock foundations. NGI report 53101-2, 67 p. (In Norwegian).

Barton, N. and Kjærnsli, B. (1981) Shear strength of rockfill. J. of the Geotech. Eng. Div., Proc. of ASCE, Vol. 107: GT7: 873-891. Proc. Paper 16374, July.

Barton, N. (1999) General report concerning some 20th Century lessons and 21st Century challenges in applied rock mechanics, safety and control of the environment. Proc. of 9th ISRM Congress, Paris, 3: 1659-1679, Balkema, Netherlands.

Leps, T.M. (1970) Review of the shearing strength of rockfill. J. of Soil Mech. and Found. Div., ASCE, Vol.96, No. SM4, Proc. Paper 7394, July 1970, 1159-1170.

Linero, S. and Palma, C. (2006) Caracterización geotecnica de materiales estériles para diseño de depósitos mineros de gran altura. (Geotechnical characterization of rock materials for design of high rock dumps). Proc. of VI th South American Rock Mechanics Congress., Cartagena, Colombia.

Marsal, R.J. (1973) Mechanical properties of rockfill. Embankment-Dam Engineering, Casagrande Volume, eds. Hirschfeld and Poulos, J. Wiley and Sons, New York, pp. 109-200.

Strøm, E. (1974), (1975) and (1978) NGI internal reports on triaxial testing of rockfill for various dams in Norway. 